\title{
THE FIFTH AND SEVENTH ORDER MOCK THETA FUNCTIONS
}

\author{
BY
}

GEORGE E. ANDREWS ${ }^{1}$

\begin{abstract}
The theory of Bailey chains is extended to yield identities for Hecke type modular forms and related generalizations. The extended results allow us to produce Hecke type series for the fifth and seventh order mock theta functions. New results on the generating function for sums of three squares also follow, and a new proof that every integer is the sum of three triangular numbers is given.
\end{abstract}

1. Introduction. The mock theta functions were named and first studied by $\mathrm{S}$. Ramanujan. Four months before he died, he sketched his results in a letter to G. H. Hardy [14, pp. 354-355]. The results in this letter formed the basis on which the intermittent study of these functions has proceeded [1-3, 11, 19, 20]. Ramanujan included in his letter [14, pp. 354-355] four separate classes of mock theta functions: one class of third order, two of fifth order, and one of seventh order.

There have been a number of deep results obtained about the third order functions [3, 11, 19]. This is because Watson was able to find representations of them which allowed him to study their behaviour under the fundamental transformations of the modular group [19]. For example,

$$
f(q)=1+\sum_{n=1}^{\infty} \frac{q^{n^{2}}}{(1+q)^{2}\left(1+q^{2}\right)^{2} \cdots\left(1+q^{n}\right)^{2}}
$$

is one of the third order functions. Watson showed that

$$
f(q) \prod_{n=1}^{\infty}\left(1-q^{n}\right)=1+4 \sum_{n=1}^{\infty} \frac{(-1)^{n} q^{n(3 n+1) / 2}}{1+q^{n}},
$$

and he then adroitly used the Poisson summation formula on the right-hand side of (1.2) to obtain the modular transformations of $f(q)$.

In 1976, Ramanujan's "Lost" Notebook came to light [5]. In it we find many results for the mock theta functions beyond those contained in Ramanujan's last letter. In particular, while (1.2) was not included in the last letter, it and generalizations of it are found in the "Lost" Notebook [5, p. 97, (3.3)].

However the fifth and seventh order functions have been more of a problem. No formula like (1.2) has ever been found for any of them, and none exists in Ramanujan's "Lost" Notebook. Indeed in his paper on the fifth order functions, Watson states [20, p. 274]: "I have failed to construct a complete and exact

Received by the editors August 6, 1984 and, in revised form, December 10, 1984.

1980 Mathematics Subject Classification. Primary 33A30, 10J05, 33A25, 05A19.

${ }^{1}$ Partially supported by National Science Foundation Grant MCS 8201733. 
transformation theory of the functions, on the lines of the transformation theory of the functions of the third order, and, in view of the complexity of all the series which are involved, I am becoming somewhat skeptical concerning the existence of an exact transformation theory for functions of the fifth order."

The object of this paper is to provide the counterparts of (1.2) for the fifth and seventh order mock theta functions. This is, I believe, the necessary first step in finding the transformation theory whose existence is doubted by Watson. As an example, let us consider

$$
f_{0}(q)=1+\sum_{n=1}^{\infty} \frac{q^{n^{2}}}{(1+q)\left(1+q^{2}\right) \cdots\left(1+q^{n}\right)},
$$

one of the fifth order mock theta functions [20, p. 277]. We shall show in $\S 5$ that

$$
f_{0}(q) \prod_{n=1}^{\infty}\left(1-q^{n}\right)=\sum_{j=-\infty}^{\infty} \sum_{n \geq|j|}(-1)^{j} q^{n(5 n+1) / 2-j^{2}}\left(1-q^{4 n+2}\right) .
$$

Note the resemblance of the expression on the right-hand side of (1.4) to certain identities for modular forms due to Hecke [12] and Rogers [15] (see §4). Presumably this resemblance can be exploited to obtain the transformation theory alluded to by Watson.

The next three sections describe the necessary background for our work. $\S 5$ is a slight digression since we are able to prove certain Hecke type identities directly from our work as well as a formula related to sums of three squares. Also we obtain the new identity

$$
\begin{aligned}
\left(\sum_{n=0}^{\infty} q^{\left(\begin{array}{c}
n+1 \\
2
\end{array}\right)}\right)^{3} & =\sum_{n=0}^{\infty} \sum_{j=0}^{2 n} \frac{q^{2 n^{2}+2 n-\left(\begin{array}{c}
j+1 \\
2
\end{array}\right)}\left(1+q^{2 n+1}\right)}{\left(1-q^{2 n+1}\right)} \\
& =\sum_{n=0}^{\infty} \sum_{j=0}^{2 n} \frac{q^{n+j(4 n+1-j) / 2}\left(1+q^{2 n+1}\right)}{\left(1-q^{2 n+1}\right)}
\end{aligned}
$$

from which follows immediately Gauss's classic result that every natural number is the sum of three triangular numbers. $\S \S 6$ and 7 contain our main results on the mock theta functions.

2. Bailey chains. In [8], we presented a comprehensive treatment of RogersRamanujan type identities based on a little known result of W. N. Bailey [9, §4]. For the statement of Bailey's Lemma we need the following standard notation:

$$
\begin{gathered}
(a ; q)_{\infty}=(a)_{\infty}=\prod_{n=0}^{\infty}\left(1-a q^{n}\right), \\
(a ; q)_{n}=(a)_{n}=(a ; q)_{\infty} /\left(a q^{n} ; q\right)_{\infty} \\
\left(=(1-a)(1-a q) \cdots\left(1-a q^{n-1}\right) \text { for } n \text { a nonnegative integer }\right) .
\end{gathered}
$$

BAILEY'S LEMMA. If for $n \geq 0$ the sequences $\left\{\alpha_{n}\right\}$ and $\left\{\beta_{n}\right\}$ are related by

$$
\beta_{n}=\sum_{r=0}^{n} \frac{\alpha_{r}}{(q)_{n-r}(a q)_{n+r}},
$$


then for $n \geq 0$

$$
\beta_{n}^{\prime}=\sum_{r=0}^{n} \frac{\alpha_{r}^{\prime}}{(q)_{n-r}(a q)_{n+r}}
$$

where

$$
\beta_{n}^{\prime}=\frac{1}{\left(a q / \rho_{1}\right)_{n}\left(a q / \rho_{2}\right)_{n}} \sum_{j=0}^{n} \frac{\left(\rho_{1}\right)_{j}\left(\rho_{2}\right)_{j}\left(a q / \rho_{1} \rho_{2}\right)_{n-j}\left(a q / \rho_{1} \rho_{2}\right)^{j} \beta_{j}}{(q)_{n-j}}
$$

and

$$
\alpha_{r}^{\prime}=\frac{\left(\rho_{1}\right)_{r}\left(\rho_{2}\right)_{r}\left(a q / \rho_{1} \rho_{2}\right)^{r} \alpha_{r}}{\left(a q / \rho_{1}\right)_{r}\left(a q / \rho_{2}\right)_{r}}
$$

The above formulation is not at all the way Bailey stated this result [9, §4]. However, formulated as above it turns out to be incredibly powerful in obtaining and understanding Rogers-Ramanujan type identities. Pairs $\alpha_{n}, \beta_{n}$ can be substituted into identities like (3.1) to yield directly Rogers-Ramanujan type identities. The point is that once you find a pair of sequences $\alpha_{n}, \beta_{n}$ that satisfies (2.3) you can produce a new pair $\alpha_{n}^{\prime}, \beta_{n}^{\prime}$ that satisfies the same identity. Thus an infinite family

$$
\left(\alpha_{n}, \beta_{n}\right) \rightarrow\left(\alpha_{n}^{\prime}, \beta_{n}^{\prime}\right) \rightarrow\left(\alpha_{n}^{\prime \prime}, \beta_{n}^{\prime \prime}\right) \rightarrow \cdots
$$

of such "Bailey pairs" can be obtained merely by iterating Bailey's Lemma. Furthermore, if only the $\alpha_{n}$ sequence is given, then the $\beta_{n}$ sequence is completely determined by (2.3). If only the $\beta_{n}$ sequence is given, then (2.3) may be inverted to yield [5, Lemma 3]

$$
\alpha_{n}=\left(1-a q^{2 n}\right) \sum_{j=0}^{n} \frac{(a q)_{n+j-1}(-1)^{n-j} q^{\left(\frac{n-j}{2}\right)}}{(q)_{n-j}} \beta_{j} .
$$

Thus if only the $\beta_{n}$ sequence is given, then the $\alpha_{n}$ sequence is completely determined by (2.7).

Furthermore, the sequence may be extended to the left as well:

$$
\cdots \rightarrow\left(\alpha_{n}^{(-2)}, \beta_{n}^{(-2)}\right) \rightarrow\left(\alpha_{n}^{(-1)}, \beta_{n}^{(-1)}\right) \rightarrow\left(\alpha_{n}, \beta_{n}\right) \rightarrow\left(\alpha_{n}, \beta_{n}^{\prime}\right) \rightarrow \cdots .
$$

Obviously from (2.6)

$$
\alpha_{r}^{(-1)}=\frac{\left(a q / \rho_{1}\right)_{r}\left(a q / \rho_{2}\right)_{r}\left(\rho_{1} \rho_{2} / a q\right)^{r} \alpha_{r}}{\left(\rho_{1}\right)_{r}\left(\rho_{2}\right)_{r}} .
$$

To back up in the chain of $\beta$ 's is a little trickier. The relation is

$$
\beta_{n}^{(-1)}=\frac{1}{\left(\rho_{1}\right)_{n}\left(\rho_{2}\right)_{n}} \sum_{j=0}^{n} \frac{\left(a q / \rho_{1}\right)_{j}\left(a q / \rho_{2}\right)_{j}\left(\rho_{1} \rho_{2} / a q\right)_{n-j}\left(\rho_{1} \rho_{2} / a j\right)^{2 n-j} \beta_{j}}{(q)_{n-j}}
$$

To see this, let us define

$$
B_{n}=\left(\frac{a q}{\rho_{1}}\right)_{n}\left(\frac{a q}{\rho_{2}}\right)_{n} \beta_{n},
$$




$$
\begin{gathered}
B_{n}^{(-1)}=\left(\rho_{1}\right)_{n}\left(\rho_{2}\right)_{n}\left(\frac{a q}{\rho_{1} \rho_{2}}\right)^{n} \beta_{n}^{(-1)}, \\
b(t)=\sum_{n=0}^{\infty} B_{n} t^{n}
\end{gathered}
$$

and

$$
b_{-1}(t)=\sum_{n=0}^{\infty} B_{n}^{(-1)} t^{n} .
$$

Then by the $q$-binomial series [4, p. 17, (2.2.1)], we see that $(2.5)$ is equivalent to

$$
b(t)=\frac{\left(a q t / \rho_{1} \rho_{2}\right)_{\infty} b_{(-1)}(t)}{(t)_{\infty}} .
$$

Obviously (2.14) is equivalent to

$$
b_{-1}(t)=\frac{(t)_{\infty}}{\left(a q t / \rho_{1} \rho_{2}\right)_{\infty}} b(t),
$$

and (2.15) yields (2.8) by invocation again of the $q$-binomial series.

We now have the four identities $((2.3),(2.7)-(2.9))$ necessary so that we may start with either sequence of a Bailey pair, obtain the other sequence and then move either direction in the Bailey chain.

3. The role of SCRATCHPAD. How can one gain a foothold in studying the mock theta functions? Our approach was to implement the study of Bailey chains on SCRATCHPAD, IBM's symbolic algebra package. To keep things as simple as possible we considered the case where $\rho_{1}$ and $\rho_{2} \rightarrow \infty$ and $a=1$. Thus from Bailey's Lemma, we see that

$$
\sum_{n=0}^{\infty} q^{n^{2}} \beta_{n}=(q)_{\infty} \sum_{n=0}^{\infty} q^{n^{2}} \alpha_{n}
$$

where by (2.7)

$$
\alpha_{n}=\left(1-q^{2 n}\right) \sum_{j=0}^{n} \frac{(q)_{n+j-1}(-1)^{n-j} q^{\left(^{n-j}\right)} \beta_{j}}{(q)_{n-j}} .
$$

Suppose we want the left side of (3.1) to coincide with (1.1). Then we must take

$$
\beta_{n}=\frac{1}{(1+q)^{2}\left(1+q^{2}\right)^{2} \cdots\left(1+q^{n}\right)^{2}} .
$$

We now define $\alpha_{n}$ and $\beta_{n}$ explicitly in SCRATCHPAD and ask for the first four values of $\alpha_{n}$. SCRATCHPAD responds with

$$
\begin{gathered}
1 \\
\frac{-4 q}{q+1} \\
\frac{4 q^{3}}{q^{2}+1} \\
\frac{-4 q^{6}}{q^{3}+1}
\end{gathered}
$$


The pattern suggested is quite clear; namely it is reasonable to conjecture that $\alpha_{n}$ is $(-1)^{n} 4 q^{n(n+1) / 2} /\left(1+q^{n}\right)$ for $n \geq 1, \alpha_{0}=1$. The insertion of this conjecture in (3.1) coincides, not surprisingly, with the known fact (1.2).

Suppose now we redefine $\beta_{n}$ by

$$
\beta_{n}=\frac{1}{(1+q)\left(1+q^{2}\right) \cdots\left(1+q^{n}\right)} .
$$

This then makes the left-side of (3.1) identical with the series in (1.3) for the fifth order mock theta function $f_{0}(q)$. The pattern for the related $\alpha_{n}$ arising from (3.2) is now somewhat complicated so we ask SCRATCHPAD for the first nine values:

$$
\begin{aligned}
& 1 \\
& q^{2}-3 q \\
& q^{7}-2 q^{6}-q^{5}+2 q^{4}+2 q^{3} \\
& q^{15}-2 q^{14}-q^{12}+4 q^{11}-2 q^{8}-2 q^{6} \\
& q^{26}-2 q^{25}+q^{22}+2 q^{21}-2 q^{18}-2 q^{17}+2 q^{13}+2 q^{10} \\
& \vdots \\
& q^{100}-2 q^{99}+2 q^{96}-q^{92}-2 q^{88}+2 q^{84}+2 q^{83} \\
& \quad-2 q^{76}-2 q^{75}+2 q^{67}+2 q^{64}-2 q^{56}-2 q^{51}+2 q^{43}+2 q^{36} .
\end{aligned}
$$

A careful look at $\alpha_{8}$ (and, in the actual discovery, $\alpha_{7}$ and $\alpha_{9}$ ) suggests that a reasonable conjecture for $\alpha_{n}$ is

$$
q^{n(3 n+1) / 2} \sum_{j=-n}^{n}(-1)^{j} q^{-j^{2}}-q^{n(3 n-1) / 2} \sum_{j=-n+1}^{n-1}(-1)^{j} q^{-j^{2}}
$$

If we insert this conjectured formula for $\alpha_{n}$ into (3.1) we obtain (1.4) almost directly. Thus SCRATCHPAD provides a powerful tool for the empirical analysis of Bailey chains. It should be emphasized that identity (1.4) appears nowhere in any of Ramanujan's known writings.

4. Extensions of Shanks' formulas. Once the truncated theta series are observed arising in (3.5), one immediately recalls the two elegant papers by D. Shanks on truncated theta series [16, 17]. Presumably, if Shanks' results can be embedded in the hierarchy of $q$-hypergeometric function identities, then adequate generalizations of his results should allow us to derive (3.5) and related formulae for the other fifth order mock theta functions. It turns out that if we move to the left one place in the Bailey chain for the fifth order mock theta functions, our work is greatly simplified. The following two lemmas provide us adequate $q$-hypergeometric series machinery for the appropriate new Bailey pairs given in Theorems 3 and 4.

Lemma 1. The sequences $A_{n}, B_{n}$ form a Bailey pair where

$$
B_{n}=\frac{(-1)^{n}(b)_{n} q^{-n(n-1) / 2} a^{-n}}{(q)_{n}(b q)_{n}}
$$




$$
A_{n}=\frac{(-1)^{n}(a q)_{n-1}\left(1-a q^{2 n}\right) q^{n(n-1) / 2}}{(b q)_{n}} \sum_{j=0}^{n} \frac{(b)_{j} q^{j(1-n)} a^{-j}}{(q)_{j}}
$$

Proof. All that is necessary is to evaluate (2.7) with $\beta_{n}$ replaced by $B_{n}$ :

$$
\begin{aligned}
A_{n} & =\left(1-a q^{2 n}\right) \sum_{j=0}^{n} \frac{(a q)_{n+j-1}(-1)^{n-j} q^{\left(\frac{n-j}{2}\right)} B_{j}}{(q)_{n-j}} \\
& =\frac{(-1)^{n}(a q)_{n-1}\left(1-a q^{2 n}\right) q^{\left(\frac{n}{2}\right)}}{(q)_{n}} \sum_{j=0}^{n} \frac{\left(a q^{n}\right)_{j}(q)_{n}(b)_{j} a^{-j} q^{j(1-n)}}{(q)_{j}(q)_{n-j}(b q)_{j}} \\
& =\frac{(-1)^{n}(a q)_{n-1}\left(1-a q^{2 n}\right) q^{\left(\frac{n}{2}\right)}}{(q)_{n}} \sum_{j=0}^{n} \frac{\left(q^{-n}\right)_{j}(b)_{j}\left(a q^{n}\right)_{j} q^{j-\left(\frac{1}{2}\right)} a^{-j}}{(q)_{j}(b q)_{j}} \\
& =\frac{(-1)^{n}(a q)_{n-1}\left(1-a q^{2 n}\right) q^{\left(\frac{n}{2}\right)}}{(q)_{n}} \lim _{t \rightarrow 0} \sum_{j=0}^{n} \frac{\left(q^{-n}\right)_{j}(b)_{j}\left(a q^{n}\right)_{j}(q / a t)^{j}}{(q)_{j}(b q)_{j}\left(t^{-1}\right)_{j}} \\
& =\frac{(-1)^{n}(a q)_{n-1}\left(1-a q^{2 n}\right) q^{\left(\frac{n}{2}\right)}}{(q)_{n}} \cdot \frac{(q)_{n}}{(b q)_{n}} \sum_{j=0}^{n} \frac{\left(q^{-n}\right)_{j}(b)_{j} a^{-j} q^{j(1-n)}}{(q)_{j}\left(q^{-n}\right)_{j}}
\end{aligned}
$$

(by [22, p. 175, (10.2)] with $a=q^{-n}, c=a q^{n}, e=b q, f=t^{-1}, p=q$ )

$$
=\frac{(-1)^{n}(a q)_{n-1}\left(1-a q^{2 n}\right) q^{\left(\frac{n}{2}\right)}}{(b q)_{n}} \sum_{j=0}^{n} \frac{(b)_{j} a^{-j} q^{j(1-n)}}{(q)_{j}}
$$

as desired.

LEMMA 2.

$$
1+\sum_{j=1}^{n} \frac{(a)_{j}\left(1-a q^{2 j}\right)(b)_{j} a^{j} q^{j^{2}} b^{-j}}{(a)_{j}(1-a)(a q / b)_{j}}=\frac{(a q)_{n}}{(a q / b)_{n}} \sum_{j=0}^{n} \frac{(b)_{j}\left(a q^{n+1} / b\right)^{j}}{(q)_{j}}
$$

Proof. This result is merely a limiting case of Watson's q-analog of Whipple's theorem [18, p. 100, (3.4.1.5)]. In fact, (4.3) follows immediately from the substitutions $g=q^{-n}, e=b, c=a q^{n+1}, f \rightarrow \infty, d \rightarrow \infty$.

We remark that if in (4.3) we take $a=1$ and let $b \rightarrow \infty$, we obtain Shank's finite version of Euler's pentagonal number theorem [16, p. 747, (2)]. Shank's finite version of Gauss's theorem [17, p. 609, $\left(3^{\prime}\right)$ ] follows by replacing $q$ by $q^{2}$ in (4.3) and then setting $a=1, b=q$.

THEOREM 3. The sequences $A_{n}, B_{n}$ form a Bailey pair where

$$
B_{n}=\frac{(-1)^{n}(b)_{n} q^{-n(n-1) / 2} a^{-n}}{(q)_{n}(b q)_{n}}
$$




$$
\begin{aligned}
A_{n}= & \frac{(-1)^{n}(a q)_{n-1}(b)_{n} a^{-n} q^{-\left(\frac{n}{2}\right)}\left(1-a q^{2 n}\right)}{(b q)_{n}(q)_{n}} \\
& +\frac{(-1)^{n} q^{\left(\frac{n}{2}\right)} b^{n-1}(a q / b)_{n-1}\left(1-a q^{2 n}\right)}{(b q)_{n}} \\
& \cdot\left(1+\sum_{j=1}^{n-1} \frac{(a q)_{j-1}\left(1-a q^{2 j}\right)(b)_{j} a^{-j} q^{-j^{2}} b^{-j}}{(q)_{j}(a q / b)_{j}}\right) .
\end{aligned}
$$

Proof. Since the $B_{n}$ sequence given by (4.4) is the same as the one in Lemma 1, equation (4.1), we see that we only need to identify the expression in (4.2) with the right-hand side of (4.5). In Lemma 2 replace $a$ by $a^{-1}, b$ by $b^{-1}, q$ by $q^{-1}$ and $n$ by $n-1$; hence

$$
\begin{aligned}
1+ & \sum_{j=1}^{n-1} \frac{(a q)_{j-1}\left(1-a q^{2 j}\right)(b)_{j} a^{-j} b^{-j} q^{-j^{2}}}{(q)_{j}(a q / b)_{j}} \\
& =\frac{b^{1-n}(a q)_{n-1}}{(a q / b)_{n-1}} \sum_{j=0}^{n-1} \frac{(b)_{j} a^{-j} q^{j(1-n)}}{(q)_{j}} .
\end{aligned}
$$

Now let us examine the sum in (4.2). We split off the term $j=n$ which yields the first summand on the right-hand side of (4.5); the remainder of the sum in (4.2) is identical with the sum of the right-hand side of (4.6). Hence multiplying both sides of (4.6) by $b^{n-1}(a q / b)_{n-1} /(a q)_{n-1}$ and substituting the resulting left-hand side into (4.2), we obtain the second term in (4.5).

THEOREM 4. The sequences $A_{n}^{\prime}, B_{n}^{\prime}$ form a Bailey pair where

$$
\begin{gathered}
B_{n}^{\prime}=\frac{1}{(b q)_{n}}, \\
A_{n}^{\prime}=\frac{(-1)^{n}(a q)_{n-1}(b)_{n} q^{\left(n_{2}^{n+1}\right)}\left(1-a q^{2 n}\right)}{(b q)_{n}(q)_{n}} \\
+\frac{(-1)^{n} a^{n} q^{n(3 n-1) / 2} b^{n-1}(a q / b)_{n-1}\left(1-a q^{2 n}\right)}{(b q)_{n}} \\
+\left(1+\sum_{j=1}^{n-1} \frac{(a q)_{j-1}\left(1-a q^{2 j}\right)(b)_{j} a^{-j} q^{-j^{2}} b^{-j}}{(q)_{j}(a q / b)_{j}}\right) .
\end{gathered}
$$

Proof. We apply Bailey's Lemma (with $\rho_{1}, \rho_{2} \rightarrow \infty$ ) to the Bailey pair in Theorem 3 and the resulting $A_{n}^{\prime}=a^{n} q^{n^{2}} A_{n}$ which is precisely (4.8). Now by (2.5)

as desired.

$$
\begin{aligned}
B_{n}^{\prime} & =\sum_{j=0}^{n} \frac{a^{j} q^{j^{2}}}{(q)_{n-j}} \frac{(-1)^{j}(b)_{j} q^{-j(j-1) / 2} a^{-j}}{(q)_{j}(b q)_{j}} \\
& =\frac{1}{(q)_{n}} \sum_{j=0}^{n} \frac{\left(q^{-n}\right)_{j}(b)_{j}}{(q)_{j}(b q)_{j}} q^{(n+1) j} \\
& =\frac{1}{(b q)_{n}} \text { by }[\mathbf{1 8}, \text { p. } 97,(3.3 .2 .6)],
\end{aligned}
$$


5. Hecke modular form identities. E. Hecke [12] made an extensive study of double theta type series involving an indefinite quadratic form. For example, he showed that

$$
(q)_{\infty}^{2}=\sum_{m=-\infty}^{\infty} \sum_{n \geq 2|m|}(-1)^{n+m} q^{\left(\begin{array}{c}
n+1 \\
2
\end{array}\right)-m(3 m-1) / 2} .
$$

Actually as D. Bressoud points out [10], identity (5.1) was discovered originally by L. J. Rogers [15]. Subsequent studies of these types of identities have been made by Kac and Peterson [13], Bressoud [10], and the author [7].

The Bailey pair in Theorem 3 leads naturally to Theorem 5, an infinite product expansion which implies several Hecke modular form type results.

THEOREM 5. Let $A_{n}$ be defined by (4.5); then

$$
\frac{(q)_{\infty}(b q / y)_{\infty}(a q)_{\infty}}{(b q)_{\infty}(q / y)_{\infty}(a q / y)_{\infty}}=\sum_{n=0}^{\infty} \frac{(y)_{n}(-a / y)^{n} q^{(n+1)} A_{n}}{(a q / y)_{n}}
$$

Proof. We take the Bailey pair from Theorem 3 and substitute into (2.4) with $\rho_{1}=y, \rho_{2} \rightarrow \infty, n \rightarrow \infty$. This yields

$$
\frac{1}{(a q / y)_{\infty}} \sum_{n=0}^{\infty} \frac{(y)_{n}(b)_{n}}{(q)_{n}(b q)_{n}}\left(\frac{q}{y}\right)^{n}=\frac{1}{(a q)_{\infty}} \sum_{r=0}^{\infty} \frac{(y)_{r}}{(a q / y)_{r}}\left(-\frac{a}{y}\right)^{r} q^{\left(r_{2}^{r+1}\right)} A_{r} .
$$

Now

$$
\sum_{n=0}^{\infty} \frac{(y)_{n}(b)_{n}(q / y)^{n}}{(q)_{n}(b q)_{n}}=\frac{(q)_{\infty}(b q / y)_{\infty}}{(b q)_{\infty}(q / y)_{\infty}}
$$

by $[18$, p. 97, (3.3.2.5)]. Substituting the right-hand side of (5.4) into (5.3) we obtain (5.2).

To apply (5.2) with ease to Hecke modular form identities, we prove three lemmas.

Lemma 6. Let $A_{n}(a, b, q)$ denote the $A_{n}$ in (4.5). Then

$$
\begin{aligned}
A_{n}(a q, b, q)= & \frac{(-1)^{n}\left(1-a q^{2 n+1}\right) q^{\left(\begin{array}{c}
n \\
2
\end{array}\right)} b^{n}(a q / b)_{n}}{(1-a q)(b q)_{n}} \\
& \cdot\left(1+\sum_{j=1}^{n} \frac{(a q)_{j-1}\left(1-a q^{2 j}\right)(b)_{j} a^{-j} q^{-j^{2}} b^{-j}}{(q)_{j}(a q / b)_{j}}\right) .
\end{aligned}
$$

Proof. By (4.2)

$$
\begin{aligned}
A_{n}(a q, b, q) & =\frac{(-1)^{n}\left(a q^{2}\right)_{n-1}\left(1-a q^{2 n+1}\right) q^{\left(\begin{array}{c}
n \\
2
\end{array}\right)}}{(b q)_{n}} \cdot \sum_{j=0}^{n} \frac{(b)_{j} q^{-j n} a^{-j}}{(q)_{j}} \\
& =\frac{(-1)^{n}(a q)_{n}\left(1-a q^{2 n+1}\right) q^{\left(\begin{array}{c}
n \\
2
\end{array}\right)}}{(1-a q)(b q)_{n}} \frac{b^{n}(a q / b)_{n}}{(a q)_{n}}
\end{aligned}
$$




$$
\begin{aligned}
& \cdot\left(1+\sum_{j=1}^{n} \frac{(a q)_{j-1}\left(1-a q^{2 j}\right)(b)_{j} a^{-j} q^{-j^{2}} b^{-j}}{(q)_{j}(a q / b)_{j}}\right) \\
= & \frac{(-1)^{n}\left(1-a q^{2 n+1}\right) q^{\left(\frac{n}{2}\right)} b^{n}(a q / b)_{n}}{(1-a q)(b q)_{n}} \\
& \cdot\left(1+\sum_{j=1}^{n} \frac{(a q)_{j-1}\left(1-a q^{2 j}\right)(b)_{j} a^{-j} q^{-j^{2}} b^{-j}}{(q)_{j}(a q / b)_{j}}\right),
\end{aligned}
$$

as desired.

Lemma 7. Let $A_{n}(a, b, q)$ denote the $A_{n}$ in (4.5). Then

$$
\begin{gathered}
A_{n}(1,-1, q)=q^{\left(\frac{n+1}{2}\right)} \sum_{j=-n}^{n}(-1)^{j} q^{-j^{2}}-q^{\left(\frac{n}{2}\right)} \sum_{j=-n+1}^{n-1}(-1)^{j} q^{-j^{2}}, \\
A_{n}(q,-1, q)=\frac{\left(1-q^{2 n+1}\right) q^{\left(\frac{n}{2}\right)}}{(1-q)}\left(1+2 \sum_{j=1}^{n}(-1)^{j} q^{-j^{2}}\right), \\
A_{n}\left(1, q^{-1}, q^{2}\right)=(-1)^{n}\left(q^{n^{2}+2 n} \sum_{j=0}^{2 n} q^{-(j+1)}-q^{n^{2}-2 n} \sum_{j=0}^{2 n-2} q^{-(j+1)}\right), \\
A_{n}\left(q^{2}, q, q^{2}\right)=\frac{(-1)^{n}\left(1+q^{2 n+1}\right) q^{n^{2}}}{1+q} \sum_{j=0}^{2 n} q^{-(j+1)}, \\
A_{n}(1,0, q)=q^{n^{2}+n} \sum_{j=-n}^{n}(-1)^{j} q^{-j(3 j+1) / 2}-q^{n^{2}-n} \sum_{j=-n+1}^{n-1}(-1)^{j} q^{-j(3 j+1) / 2}, \\
A_{n}(q, 0, q)=\frac{q^{n^{2}}\left(1-q^{2 n+1}\right)}{(1-q)} \sum_{j=-n}^{n}(-1)^{j} q^{-j(3 j+1) / 2} .
\end{gathered}
$$

Proof. By (4.5),

$$
\begin{aligned}
A_{n}(1,-1, q) & =(-1)^{n} 2 q^{-\left(\frac{n}{2}\right)}-q^{\left(\frac{n}{2}\right)}\left(1-q^{n}\right)\left(1+2 \sum_{j=1}^{n-1}(-1)^{j} q^{-j^{2}}\right) \\
& =q^{\left(\frac{n+1}{2}\right)} \sum_{j=-n}^{n}(-1)^{j} q^{-j^{2}}-q^{\left(\frac{n}{2}\right)} \sum_{j=-n+1}^{n-1}(-1)^{j} q^{-j^{2}},
\end{aligned}
$$

as asserted in (5.6).

By (5.5),

$$
A_{n}(q,-1, q)=\frac{\left(1-q^{2 n+1}\right) q^{\left(\frac{n}{2}\right)}}{(1-q)}\left(1+2 \sum_{j=1}^{n}(-1)^{j} q^{-j^{2}}\right),
$$

as asserted in (5.7). 
By (4.5),

$$
\begin{aligned}
& A_{n}\left(1, q^{-1}, q^{2}\right)=\frac{(-1)^{n}\left(1+q^{2 n}\right) q^{n-n^{2}}\left(1-q^{-1}\right)}{\left(1-q^{2 n-1}\right)}+\frac{(-1)^{n} q^{(n-1)^{2}}\left(1-q^{4 n}\right)}{(1-q)} \\
& \cdot\left(1+\sum_{j=1}^{n-1} \frac{\left(1+q^{2 j}\right)\left(1-q^{-1}\right)(1-q) q^{j-2 j^{2}}}{\left(1-q^{2 j-1}\right)\left(1-q^{2 j+1}\right)}\right) \\
& =\frac{(-1)^{n}\left(1+q^{2 n}\right) q^{n-n^{2}}\left(1-q^{-1}\right)}{\left(1-q^{2 n-1}\right)} \\
& +\frac{(-1)^{n} q^{(n-1)^{2}}\left(1-q^{4 n}\right)}{(1-q)} \sum_{j=-n+1}^{n-1} \frac{\left(1-q^{-1}\right)(1-q) q^{j-2 j^{2}}}{\left(1-q^{2 j-1}\right)\left(1-q^{2 j+1}\right)} \\
& =\frac{(-1)^{n}\left(1+q^{2 n}\right) q^{n-n^{2}}\left(1-q^{-1}\right)}{\left(1-q^{2 n-1}\right)}+\frac{(-1)^{n} q^{(n-1)^{2}}\left(1-q^{4 n}\right)\left(1-q^{-1}\right)}{q^{-1}\left(1-q^{2}\right)} \\
& \cdot \sum_{j=-n+1}^{n-1} q^{j-2 j^{2}}\left(\frac{q^{-1}}{1-q^{2 j-1}}-\frac{q}{1-q^{2 j+1}}\right) \\
& =\frac{(-1)^{n}\left(1+q^{2 n}\right) q^{n-n^{2}}\left(1-q^{-1}\right)}{\left(1-q^{2 n-1}\right)} \\
& +\frac{(-1)^{n} q^{(n-1)^{2}}\left(1-q^{4 n}\right)}{(1+q)} \sum_{j=-n+1}^{n-1} \frac{q^{j-2 j^{2}}(1+q)}{\left(1-q^{2 j+1}\right)}
\end{aligned}
$$

(where we have replaced $j$ by $-j$ in the first sum)

$$
\begin{aligned}
& =\frac{(-1)^{n}\left(1+q^{2 n}\right) q^{n-n^{2}}\left(1-q^{-1}\right)}{\left(1-q^{2 n-1}\right)}-\frac{(-1)^{n} q^{(n-1)^{2}-n-2 n^{2}}\left(1-q^{4 n}\right)}{\left(1-q^{-2 n+1}\right)} \\
& +(-1)^{n} q^{(n-1)^{2}}\left(1-q^{4 n}\right) \sum_{j=0}^{n-1}\left(\frac{q^{j-2 j^{2}}}{1-q^{2 j+1}}+\frac{q^{-j-1-2(j+1)^{2}}}{1-q^{-2 j-1}}\right) \\
& =(-1)^{n} q^{-n^{2}-n}\left(1+q^{2 n}\right) \\
& -(-1)^{n} q^{(n-1)^{2}}\left(1-q^{4 n}\right) \sum_{j=0}^{n-1} \frac{q^{-2 j^{2}-3 j-2}\left(1-q^{4 j+2}\right)}{\left(1-q^{2 j+1}\right)} \\
& =(-1)^{n} q^{-n^{2}-n}\left(1+q^{2 n}\right) \\
& -(-1)^{n} q^{n^{2}-2 n}\left(1-q^{4 n}\right) \sum_{j=0}^{n-1}\left(q^{-\left({ }^{2 j+2}\right)}+q^{-\left({ }^{2 j+1}\right)}\right) \\
& =(-1)^{n} q^{-n^{2}-n}\left(1+q^{2 n}\right)-(-1)^{n} q^{n^{2}-2 n}\left(1-q^{4 n}\right) \sum_{j=0}^{2 n-1} q^{-\left(j \frac{1}{2}\right)} \\
& =(-1)^{n} q^{n^{2}+2 n} \sum_{j=0}^{2 n} q^{-(j+1)}-(-1)^{n} q^{n^{2}-2 n} \sum_{j=0}^{2 n-2} q^{-(j+1)},
\end{aligned}
$$

as asserted in (5.8). 
Next by (5.5)

$$
\begin{aligned}
A_{n}\left(q^{2}, q, q^{2}\right) & =\frac{(-1)^{n}\left(1+q^{2 n+1}\right) q^{n^{2}}}{1+q}\left(1+\sum_{j=1}^{n}\left(1+q^{2 j}\right) q^{-2 j^{2}-j}\right) \\
& =\frac{(-1)^{n}\left(1+q^{2 n+1}\right) q^{n^{2}}}{1+q} \sum_{j=0}^{2 n} q^{-\left(\frac{j}{2}\right)},
\end{aligned}
$$

as asserted in (5.9).

By (4.5),

$$
\begin{aligned}
A_{n}(1,0, q)= & (-1)^{n} q^{-\left(\begin{array}{c}
n \\
2
\end{array}\right)}\left(1+q^{n}\right) \\
& -q^{n^{2}--n}\left(1-q^{2 n}\right)\left(1+\sum_{j=1}^{n-1}\left(1+q^{j}\right)(-1)^{j} q^{-j(3 j+1) / 2}\right) \\
= & (-1)^{n} q^{-\left(\begin{array}{l}
n \\
2
\end{array}\right)}\left(1+q^{n}\right)-q^{n^{2}-n}\left(1-q^{2 n}\right) \sum_{j=-n+1}^{n-1}(-1)^{j} q^{-j(3 j+1) / 2} \\
= & q^{n^{2}+n} \sum_{j=-n}^{n}(-1)^{j} q^{-j(3 j+1) / 2}-q^{n^{2}-n} \sum_{j=-n+1}^{n-1}(-1)^{j} q^{-j(3 j+1) / 2},
\end{aligned}
$$

as asserted in (5.10).

Finally by (5.5)

$$
\begin{aligned}
A_{n}(q, 0, q) & =\frac{q^{n^{2}}\left(1-q^{2 n+1}\right)}{(1-q)}\left(1+\sum_{j=1}^{n}\left(1+q^{j}\right) q^{-j^{2}-\left(j_{2}^{j+1}\right)}(-1)^{j}\right) \\
& =\frac{q^{n^{2}}\left(1-q^{2 n+1}\right)}{(1-q)} \sum_{j=-n}^{n}(-1)^{j} q^{-j(3 j+1) / 2},
\end{aligned}
$$

which is (5.11).

Lemma 8. Let $A_{n}=A_{n}(a, b, q)$ be defined by (4.5). Then

$$
\begin{gathered}
\frac{(q)_{\infty}(a q)_{\infty}}{(b q)_{\infty}}=\sum_{n=0}^{\infty} a^{n} q^{n^{2}} A_{n} \\
\frac{(q)_{\infty}^{2}(-b q)_{\infty}}{(-q)_{\infty}^{2}(b q)_{\infty}}=1+2 \sum_{n=1}^{\infty} \frac{q^{n(n+1) / 2}}{1+q^{n}} A_{n}(1, b, q) \\
\frac{\left(q^{2} ; q^{2}\right)_{\infty}^{2}\left(b q ; q^{2}\right)_{\infty}}{\left(q ; q^{2}\right)_{\infty}^{2}\left(b q^{2} ; q^{2}\right)_{\infty}}=\sum_{n=0}^{\infty}(-1)^{n} q^{n^{2}} A_{n}\left(1, b, q^{2}\right)
\end{gathered}
$$

Proof. These identities are immediate from Theorem 5 under the substitutions $y \rightarrow \infty ; a=1, y=-1$; finally replace $q$ by $q^{2}$, then set $a=1, y=q$.

It is now possible to combine Lemmas 7 and 8 to obtain 18 different identities of 
the Hecke modular form type. We note only:

$$
\begin{aligned}
(q)_{\infty}^{2}\left(q ; q^{2}\right)_{\infty} & =1+\sum_{\substack{n=1 \\
|j| \leq n}}^{\infty}(-1)^{j} q^{n(3 n+1) / 2-j^{2}}-\sum_{\substack{n=1 \\
|j|<n}}^{\infty}(-1)^{j} q^{n(3 n-1) / 2-j^{2}} \\
& =\sum_{\substack{n=0 \\
|j| \leq n}}^{\infty}(-1)^{j} q^{n(3 n+1) / 2-j^{2}}\left(1-q^{2 n+1}\right) .
\end{aligned}
$$

This result follows from (5.12) with $a=1, b=-1$ and (5.6). We can also find a new representation for the generating function of $r_{3}(n)$, the number of representations of $n$ as a sum of three squares:

$$
\begin{aligned}
\sum_{n=0}^{\infty}(-1)^{n} r_{3}(n) q^{n} & =\left(\sum_{n=-\infty}^{\infty}(-1)^{n} q^{n^{2}}\right)^{3}=\left(\frac{(q)_{\infty}}{(-q)_{\infty}}\right)^{3} \\
& =1+4 \sum_{n=1}^{\infty} \frac{(-1)^{n} q^{n}}{1+q^{n}}-2 \sum_{\substack{n=1 \\
|j|<n}}^{\infty} \frac{q^{n^{2}-j^{2}}\left(1-q^{n}\right)(-1)^{j}}{1+q^{n}}
\end{aligned}
$$

The first two equalities are classical [4, p. 23, (2.2.12)]; the last follows from (5.13) with $b=-1$ and (5.6).

Finally we consider (5.2) in the case $q \rightarrow q^{2}$. Then $a=q^{2}, b=y=q$. This yields

$$
\begin{aligned}
& \left(\sum_{n=0}^{\infty} q^{(n+1)}\right)^{3}=\left(\frac{\left(q^{2} ; q^{2}\right)_{\infty}}{\left(q ; q^{2}\right)_{\infty}}\right)^{3} \\
& =\sum_{n=0}^{\infty} \sum_{j=0}^{2 n} \frac{q^{2 n^{2}+2 n-\left(\begin{array}{c}
j+1 \\
2
\end{array}\right)}\left(1+q^{2 n+1}\right)}{\left(1-q^{2 n+1}\right)} \\
& =\sum_{n=0}^{\infty} \sum_{j=0}^{2 n} \frac{q^{n+j(4 n+1-j) / 2}\left(1+q^{2 n+1}\right)}{\left(1-q^{2 n+1}\right)} \text {. }
\end{aligned}
$$

Clearly the left-hand side of (5.17) is the generating function for the number of representations of an integer as a sum of three triangular numbers. Inspecting the last entry in (5.7) we see that in this series the $n$th term contributes at least 1 to the coefficient of $q^{n}$; hence all coefficients are positive. Thus we have just proved Gauss's famous result that every integer is the sum of three triangular numbers.

6. The fifth order mock theta functions. In the following theorem, eight of the ten fifth order mock theta functions of Ramanujan [14, pp. 354-355] will be related to double sums of the Hecke modular form type. We are not able to give such formulas for either $\chi_{0}(q)$ or $\chi_{1}(q)$ [20, pp. 277-278]. However, since $\chi_{0}(q)=2 F_{0}(q)-\phi_{0}(-q)$ and $\chi_{1}(q)=2 F_{1}(q)+q^{-1} \phi_{1}(-q)\left[20\right.$, pp. 277, 279], we see that each of $\chi_{0}(q)$ and $\chi_{1}(q)$ is a linear combination of functions that do appear in our main result. Each equation in Theorem 9 will provide both the definition and the appropriate Hecke representation of the fifth order mock theta function under consideration. 


\section{TheOREM 9.}

$$
\begin{aligned}
f_{0}(q) \equiv \sum_{n=0}^{\infty} \frac{q^{n^{2}}}{(-q)_{n}}=\frac{1}{(q)_{\infty}} \sum_{\substack{n=0 \\
|j| \leq n}}^{\infty}(-1)^{j} q^{n(5 n+1) / 2-j^{2}}\left(1-q^{4 n+2}\right) \\
F_{0}(q) \equiv \sum_{n=0}^{\infty} \frac{q^{2 n^{2}}}{\left(q ; q^{2}\right)_{n}} \\
=\frac{1}{\left(q^{2} ; q^{2}\right)_{\infty}} \sum_{n=0}^{\infty} \sum_{j=0}^{2 n}(-1)^{n} q^{5 n^{2}+2 n-\left(\frac{j+1}{2}\right)}\left(1+q^{6 n+3}\right)
\end{aligned}
$$

(6.3) $1+2 \psi_{0}(q) \equiv \sum_{n=0}^{\infty}(-1 ; q)_{n} q^{\left(\begin{array}{c}n+1 \\ 2\end{array}\right)}$

$$
=\frac{(-q)_{\infty}}{(q)_{\infty}}\left(1+2 \sum_{n=1}^{\infty}(-1)^{n} q^{2 n^{2}+n}-2 \sum_{\substack{n=1 \\|j|<n}}^{\infty}(-1)^{j} q^{n(5 n-1) / 2-j(3 j+1) / 2}\left(1-q^{n}\right)\right)
$$

$$
\begin{aligned}
\phi_{0}(q) & \equiv \sum_{n=0}^{\infty}\left(-q ; q^{2}\right)_{n} q^{n^{2}} \\
& =\frac{\left(-q ; q^{2}\right)}{\left(q^{2} ; q^{2}\right)} \sum_{\substack{n=0 \\
|j| \leq n}}^{\infty}(-1)^{j} q^{5 n^{2}+2 n-3 j^{2}-j}\left(1-q^{6 n+3}\right),
\end{aligned}
$$

$$
f_{1}(q) \equiv \sum_{n=0}^{\infty} \frac{q^{n^{2}+n}}{(-q)_{n}}=\frac{1}{(q)_{\infty}} \sum_{\substack{n=0 \\|j| \leq n}}^{\infty}(-1)^{j} q^{n(5 n+3) / 2-j^{2}}\left(1-q^{2 n+1}\right)
$$

$$
\begin{aligned}
F_{1}(q) & =\sum_{n=0}^{\infty} \frac{q^{2 n^{2}+2 n}}{\left(q ; q^{2}\right)_{n+1}} \\
& =\frac{1}{\left(q^{2} ; q^{2}\right)_{\infty}} \sum_{n=0}^{\infty} \sum_{j=0}^{2 n}(-1)^{n} q^{5 n^{2}+4 n-\left(\begin{array}{l}
j+1 \\
2
\end{array}\right)}\left(1+q^{2 n+1}\right)
\end{aligned}
$$

$$
\begin{aligned}
\psi_{1}(q) & =\sum_{n=0}^{\infty}(-q)_{n} q^{\left(\begin{array}{c}
n+1 \\
2
\end{array}\right)} \\
& =\frac{(-q)_{\infty}}{(q)_{\infty}} \sum_{\substack{n=0 \\
|j| \leq n}}^{\infty}(-1)^{j} q^{n(5 n+3) / 2-j(3 j+1) / 2}\left(1-q^{2 n+1}\right),
\end{aligned}
$$

$$
\begin{aligned}
\phi_{1}(q) & =\sum_{n=0}^{\infty}\left(-q ; q^{2}\right)_{n} q^{(n+1)^{2}} \\
& =\frac{\left(-q ; q^{2}\right)_{\infty}}{\left(q^{2} ; q^{2}\right)_{\infty}} \sum_{\substack{n=0 \\
|j| \leq n}}^{\infty}(-1)^{j} q^{5 n^{2}+4 n-3 j^{2}-j}\left(1+q^{2 n+1}\right)
\end{aligned}
$$

Proof. We shall rely on many of the results in $\S \S 4$ and 5 . Our jumping off point 
is Theorem 4 and the observation that $A_{n}^{\prime}(a, b, q)=a^{n} q^{n^{2}} A_{n}(a, b, q)$. Thus all our formulae for the $A_{n}(a, b, q)$ can be applied in evaluating $A_{n}^{\prime}(a, b, q)$.

We begin with (6.1), and let $B_{n}^{\prime}=B_{n}^{\prime}(b, q)$.

$$
\begin{aligned}
f_{0}(q) & =\sum_{n=0}^{\infty} q^{n^{2}} B_{n}^{\prime}(-1, q) \quad(\text { by }(4.7)) \\
& =\frac{1}{(q)_{\infty}} \sum_{n=0}^{\infty} q^{n^{2}} A_{n}^{\prime}(1,-1, q) \quad(\text { by }(3.1)) \\
& =\frac{1}{(q)_{\infty}} \sum_{n=0}^{\infty} q^{2 n^{2}} A_{n}(1,-1, q) \quad(\text { by }(2.6)) \\
& =\frac{1}{(q)_{\infty}}\left(\sum_{\substack{n=0 \\
|j| \leq n}}^{\infty} q^{n(5 n+1) / 2-j^{2}}(-1)^{j}-\sum_{\substack{n=0 \\
|j|<n}}^{\infty} q^{n(5 n-1) / 2-j^{2}}(-1)^{j}\right) \\
& =\frac{1}{(q)_{\infty}} \sum_{\substack{n=0 \\
|j| \leq n}}^{\infty} q^{n(5 n+1) / 2-j^{2}}(-1)^{j}\left(1-q^{4 n+2}\right),
\end{aligned}
$$

which is (6.1).

Next

$$
\begin{aligned}
F_{0}(q) & =\sum_{n=0}^{\infty} q^{2 n^{2}} B_{n}^{\prime}\left(q^{-1}, q^{2}\right) \quad(\text { by }(4.7)) \\
& =\frac{1}{\left(q^{2} ; q^{2}\right)_{\infty}} \sum_{n=0}^{\infty} q^{2 n^{2}} A_{n}^{\prime}\left(1, q^{-1}, q^{2}\right) \quad(\text { by }(3.1)) \\
& =\frac{1}{\left(q^{2} ; q^{2}\right)} \sum_{n=0}^{\infty} q^{4 n^{2}} A_{n}\left(1, q^{-1}, q^{2}\right) \quad(\text { by }(2.6)) \\
& \left.\left.=\frac{1}{\left(q^{2} ; q^{2}\right)_{\infty}}\left(\sum_{n=0}^{\infty} \sum_{j=0}^{2 n}(-1)^{n} q^{5 n^{2}+2 n-\left(\frac{j}{2}\right)}\right) \quad-\sum_{n=0}^{\infty} \sum_{j=0}^{2 n-2}(-1)^{n} q^{5 n^{2}-2 n-j^{j+1}}\right)\right) \\
& =\frac{1}{\left(q^{2} ; q^{2}\right)} \sum_{n=0}^{\infty} \sum_{j=0}^{2 n}(-1)^{n} q^{5 n^{2}+2 n-\left(_{2}^{j+1}\right)}\left(1+q^{6 n+3}\right),
\end{aligned}
$$

as asserted in (6.2).

$$
\begin{aligned}
& 1+2 \psi_{0}(q)\left.=\sum_{n=0}^{\infty}(-1 ; q)_{n} q^{\left({ }_{2}+1\right.}\right) B_{n}^{\prime}(0, q) \\
&=\frac{(-q)_{\infty}}{(q)_{\infty}} \sum_{n=0}^{\infty} \frac{(-1 ; q)_{n} q^{\left({ }_{2}^{n+1}\right)} A_{n}^{\prime}(1,0, q)}{(-q ; q)_{n}} \\
& \quad\left(\text { by }(2.4), n \rightarrow \infty, \rho_{1} \rightarrow \infty, \rho_{2}=-1, a=1\right)
\end{aligned}
$$




$$
\begin{aligned}
& =\frac{(-q)_{\infty}}{(q)_{\infty}}\left(1+2 \sum_{n=1}^{\infty} \frac{q^{(n+1)+n_{2}^{2}}}{1+q^{n}} A_{n}(1,0, q)\right) \\
& =\frac{(-q)_{\infty}}{(q)_{\infty}}\left(1+2 \sum_{n=1}^{\infty} \frac{q^{n(3 n+1) / 2}}{1+q^{n}}\left((-1)^{n} q^{\left(\begin{array}{c}
n+1 \\
2
\end{array}\right)}\left(1+q^{n}\right)\right.\right. \\
& \left.\left.-q^{n^{2}-n}\left(1-q^{2 n}\right) \sum_{|j|<n}(-1)^{j} q^{-j(3 j+1) / 2}\right)\right) \\
& \text { (by (5.10)) } \\
& =\frac{(-q)_{\infty}}{(q)_{\infty}} \mid 1+2 \sum_{n=1}^{\infty}(-1)^{n} q^{2 n^{2}+n} \\
& \left.-2 \sum_{\substack{n=1 \\
|j|<n}}^{\infty}(-1)^{j} q^{n(5 n-1) / 2-j(3 j+1) / 2}\left(1-q^{n}\right)\right),
\end{aligned}
$$

as desired.

Next

$$
\begin{aligned}
\phi_{0}(q) & =\sum_{n=0}^{\infty} q^{n^{2}}\left(-q ; q^{2}\right)_{n} B_{n}^{\prime}\left(0, q^{2}\right) \\
& =\frac{\left(-q ; q^{2}\right)_{\infty}}{\left(q^{2} ; q^{2}\right)_{\infty}} \sum_{n=0}^{\infty} q^{n^{2}} A_{n}^{\prime}\left(1,0, q^{2}\right) \\
& =\frac{\left(-q ; q^{2}\right)_{\infty}}{\left(q^{2} ; q^{2}\right)_{\infty}} \sum_{n=0}^{\infty} q^{3 n^{2}} A_{n}\left(1,0, q^{2}\right) \quad\left(\text { by }(2.4), q \rightarrow q^{2}, n \rightarrow \infty, \rho_{1} \rightarrow \infty, \rho_{2}=-q, a=1\right) \\
& =\frac{\left(-q ; q^{2}\right)_{\infty}}{\left(q^{2} ; q^{2}\right)_{\infty}} \sum_{n=0}^{\infty} q^{3 n^{2}}\left(q^{2 n^{2}+2 n} \sum_{|j| \leq n}(-1)^{j} q^{-3 j^{2}-j}\right. \\
& \left.=\frac{\left(-q ; q^{2}\right)_{\infty}}{\left(q^{2} ; q^{2}\right)_{\infty}} \sum_{\substack{n=0 \\
|j| \leq n}}^{\infty}(-1)^{j} q^{5 n^{2}-2 n} \sum_{|j|<n}(-1)^{j} q^{-3 j^{2}-j}\right)
\end{aligned}
$$

as asserted in (6.4).

The proofs of the remaining four identities in Theorem 9 are exactly parallel to the four just given. The fundamental change concerns the replacement of $a$ by $q$ (or by $q^{2}$ when $q$ has been replaced by $q^{2}$ ). We therefore omit the details.

7. The seventh order mock theta functions. These three functions are perhaps the most mysterious in all of Ramanujan's work. They are defined in the last three lines of Ramanujan's Collected Papers [14, p. 355]. His only assertion about them is that 
they are not related to each other. G. N. Watson makes cryptic reference to them in $[19$, p. 80]; his short paper on the dilogarithm [21] was clearly motivated by a less than successful study of the asymptotics of the seventh order functions as $q \rightarrow 1^{-}$. To my knowledge nothing else about them appears in the literature.

We are able to produce a counterpart of Theorem 9 for them; however the scope of our results is much more limited than the generality of results related to the fifth order functions. Clearly much remains unknown about the seventh order functions; I hope that these results will facilitate further study.

Our work relies on the Bailey chain theory of $\$ 2$. We begin with the lemmas necessary to establish the appropriate Bailey pairs. The polynomials to be considered are best expressed in terms of the Gaussian polynomials [4, p. 35]:

$$
\left[\begin{array}{c}
n \\
m
\end{array}\right]=\left[\begin{array}{c}
n \\
m
\end{array}\right]_{q}=\frac{(q)_{n}}{(q)_{m}(q)_{n-m}}
$$

These polynomials satisfy the recurrences $[4$, p. 35]:

$$
\begin{aligned}
{\left[\begin{array}{l}
n \\
m
\end{array}\right] } & =\left[\begin{array}{c}
n-1 \\
m-1
\end{array}\right]+q^{m}\left[\begin{array}{c}
n-1 \\
m
\end{array}\right] \\
& =\left[\begin{array}{c}
n-1 \\
m
\end{array}\right]+q^{n-m}\left[\begin{array}{c}
n-1 \\
m-1
\end{array}\right] .
\end{aligned}
$$

LEMMA 10. Let sequences of polynomials (in q) $U_{n}, V_{n}, W_{n}$ be defined by

$$
\begin{gathered}
U_{n}=\sum_{j=1}^{n}\left[\begin{array}{c}
n+j-1 \\
2 j-1
\end{array}\right](q)_{j-1}(-1)^{n-j} q^{\left(\frac{n-j}{2}\right)}, \\
V_{n}=\left(1-q^{2 n}\right) \sum_{j=0}^{n}\left[\begin{array}{l}
n+j \\
n-j
\end{array}\right] \frac{(q)_{j}}{1-q^{n+j}}(-1)^{n-j} q^{\left(\frac{n-j}{2}\right)}, \\
W_{n}=\sum_{j=0}^{n}\left[\begin{array}{l}
n+j \\
n-j
\end{array}\right](q)_{j}(-1)^{n-j} q^{\left(\frac{n-j}{2}\right)} .
\end{gathered}
$$

Then

$$
\begin{gathered}
V_{n}=W_{n}-q^{2 n-1} W_{n-1}, \\
V_{n}=-\left(1-q^{n}\right) q^{2 n-2} U_{n-1}+(-1)^{n} 2 q^{\left(_{2}^{(n+1)}\right)}, \\
W_{n}=-q^{n} U_{n}-q^{2 n-2} U_{n-1} .
\end{gathered}
$$

Proof. Since $1-q^{2 n}=1-q^{n+j}+q^{n+j}\left(1-q^{n-j}\right)$, we may rewrite $V_{n}$ as

$$
\begin{aligned}
V_{n} & =\sum_{j=0}^{n}\left(\left[\begin{array}{l}
n+j \\
n-j
\end{array}\right]+q^{n+j}\left[\begin{array}{l}
n+j-1 \\
n-j-1
\end{array}\right]\right)(q)_{j}(-1)^{n-j} q^{\left(\frac{n-j}{2}\right)} \\
& =W_{n}-q^{2 n-1} \sum_{j=0}^{n-1}\left[\begin{array}{l}
n-1+j \\
n-1-j
\end{array}\right](q)_{j}(-1)^{n-1-j} q^{\left(\frac{n-1-j}{2}\right)} \\
& =W_{n}-q^{2 n-1} W_{n-1},
\end{aligned}
$$

which is (7.7). 


$$
\begin{aligned}
& \frac{V_{n}-(-1)^{n} 2 q^{\left(\begin{array}{c}
n+1 \\
2
\end{array}\right)}}{1-q^{n}}=(-1)^{n} q^{\left(\frac{n}{2}\right)}+\sum_{j=1}^{n}\left[\begin{array}{l}
n+j \\
n-j
\end{array}\right] \frac{\left(1+q^{n}\right)}{\left(1-q^{n-j}\right)}(q)_{j}(-1)^{n-j} q^{\left(\begin{array}{c}
n-j \\
2
\end{array}\right)} \\
& =(-1)^{n} q^{\left(\frac{n}{2}\right)}+\sum_{j=1}^{n}\left[\begin{array}{l}
n+j-1 \\
n-j-1
\end{array}\right](q)_{j}(-1)^{n-j} q^{\left(\frac{n-j}{2}\right)} \\
& +\sum_{j=1}^{n}\left[\begin{array}{c}
n+j-1 \\
n-1
\end{array}\right](q)_{j-1}(-1)^{n-j} q^{\left(\frac{n-j+1}{2}\right)} \\
& \text { (where we have used } 1+q^{n}=\left(1-q^{n-j}\right)+q^{n-j}\left(1+q^{j}\right) \text { ) } \\
& =\sum_{j=0}^{n}\left[\begin{array}{l}
n+j-1 \\
n-j-1
\end{array}\right](q)_{j}(-1)^{n-j} q^{\left(\frac{n-j}{2}\right)} \\
& -\sum_{j=0}^{n-1}\left[\begin{array}{c}
n+j \\
n-j-1
\end{array}\right](q)_{j}(-1)^{n-j} q^{(n-j)} \\
& =-\sum_{j=0}^{n-2} q^{2 j+1}\left[\begin{array}{l}
n+j-1 \\
n-j-2
\end{array}\right](q)_{j}(-1)^{n-j} q^{\left(n_{2}^{n-j}\right)} \quad(\text { by }(7.3)) \\
& =-q^{2 n-2} \sum_{j=1}^{n-1}\left[\begin{array}{c}
n-1+j-1 \\
n-1-j
\end{array}\right](q)_{j-1}(-1)^{n-j-1} q^{(n-1-j)} \\
& =-q^{2 n-2} U_{n-1} \text {, }
\end{aligned}
$$

which is equivalent to (7.8).

Finally,

$$
\begin{aligned}
W_{n}+ & q^{n} U_{n}=\sum_{j \geq 0}\left[\begin{array}{c}
n+j \\
n-j
\end{array}\right](q)_{j}(-1)^{n-j} q^{\left(\frac{n}{2}{ }^{j}\right)} \\
& +q^{n} \sum_{j=1}^{n}\left[\begin{array}{c}
n+j-1 \\
n-j
\end{array}\right](q)_{j-1}(-1)^{n-j} q^{\left(\frac{n-j}{2}\right)} \\
= & (-1)^{n} q^{\left(\frac{n}{2}\right)}+\sum_{j=1}^{n}(q)_{j-1}(-1)^{n-j} q^{\left(\frac{n-j}{2}\right)}\left(\left[\begin{array}{c}
n+j \\
n-j
\end{array}\right]\left(1-q^{j}\right)+q^{n}\left[\begin{array}{c}
n+j-1 \\
n-j
\end{array}\right]\right) \\
= & (-1)^{n} q^{\left(\frac{n}{2}\right)}+\left(1+q^{n}\right) \sum_{j=1}^{n}(q)_{j}(-1)^{n-j} q^{\left(\frac{n-j}{2}\right)} \frac{(q)_{n+j-1}}{(q)_{2 j}(q)_{n-j}} \\
= & (-1)^{n} q^{\left(\frac{n}{2}\right)}+\sum_{j \geq 1}(q)_{j}(-1)^{n-j} q^{\left(\frac{n-j}{2}\right)}\left[\begin{array}{c}
n+j-1 \\
n-j-1
\end{array}\right] \\
& +\sum_{j \geq 1}(q)_{j-1}(-1)^{n-j} q^{\left(\frac{n-j+1}{2}\right)}\left[\begin{array}{c}
n+j-1 \\
n-j
\end{array}\right]
\end{aligned}
$$

(where we have used $\left.1+q^{n}=\left(1-q^{n-j}\right)+q^{n-j}\left(1+q^{j}\right)\right)$ 


$$
\begin{aligned}
= & \sum_{j \geq 0}(q)_{j}(-1)^{n-j} q^{\left(\frac{n-j}{2}\right)}\left[\begin{array}{l}
n+j-1 \\
n-j-1
\end{array}\right] \\
& +\sum_{j \geq 1}(q)_{j-1}(-1)^{n-j} q^{\left(\frac{n-j+1}{2}\right)}\left(q^{2 j-1}\left[\begin{array}{l}
n+j-2 \\
n-j-1
\end{array}\right]+\left[\begin{array}{c}
n+j-2 \\
n-j
\end{array}\right]\right) \\
= & \sum_{j=0}^{n-1}(q)_{j}(-1)^{n-j} q^{\left(\frac{n-j}{2}\right)}\left[\begin{array}{c}
n+j-1 \\
n-j-1
\end{array}\right]-q^{2 n-2} U_{n-1} \\
& +\sum_{j=1}^{n}(q)_{j-1}(-1)^{n-j} q^{(n-j+1)}\left[\begin{array}{c}
n+j-2 \\
n-j
\end{array}\right] \\
= & -q^{2 n-2} U_{n-1},
\end{aligned}
$$

since the third term is the same as the first once $j$ is replaced by $j+1$. Thus (7.9) is estabished.

LEMMA 11. For the $U_{n}$ defined by (7.4) we have

$$
\begin{gathered}
U_{2 n}=-2 q^{3 n^{2}-2 n} \sum_{j=0}^{n-1} q^{-j^{2}-j} \\
U_{2 n+1}=q^{3 n^{2}+n} \sum_{|j| \leq n} q^{-j^{2}}
\end{gathered}
$$

Proof. Equations (7.10) and (7.11) follow directly by induction from the initial values $U_{0}=0, U_{1}=1, U_{2}=-2 q$, and for $n \geq 3$

$$
U_{n}-q^{3 n-5} U_{n-2}=-(-1)^{n} 2 q^{\left(\begin{array}{c}
n \\
2
\end{array}\right)} .
$$

The initial values follow immediately from (7.4). Now by (7.7)-(7.9)

$$
\begin{aligned}
& \left(-q^{n} U_{n}-q^{2 n-2} U_{n-1}\right)-q^{2 n-1}\left(-q^{n-1} U_{n-1}-q^{2 n-4} U_{n-2}\right) \\
& =W_{n}-q^{2 n-1} W_{n-1}=V_{n}=-\left(1-q^{n}\right) q^{2 n-2} U_{n-1}+(-1)^{n} 2 q^{\left(\begin{array}{c}
n+1 \\
2
\end{array}\right)}
\end{aligned}
$$

simplifying the extremes of this equation we find

$$
-q^{n} U_{n}+q^{4 n-5} U_{n-2}=(-1)^{n} 2 q^{\left(\begin{array}{c}
n+1 \\
2
\end{array}\right)},
$$

and multiplying through by $-q^{-n}$ we obtain (7.12).

We are now prepared to derive the Bailey pairs for the seventh order mock theta functions.

Define

$$
\begin{gathered}
\mathscr{B}_{n}(0)=\frac{1}{\left(q^{n+1}\right)_{n}}, \\
\mathscr{B}_{n}(1)=\frac{1}{\left(q^{n}\right)_{n}}, \quad n>0, \quad \mathscr{B}_{0}(1)=0 \\
\mathscr{B}_{n}(2)=\frac{1}{\left(q^{n+1}\right)_{n+1}},
\end{gathered}
$$


and

$$
\begin{gathered}
\mathscr{A}_{2 n}(0)=q^{3 n^{2}+n} \sum_{|j| \leq n} q^{-j^{2}}-q^{3 n^{2}-n} \sum_{|j|<n} q^{-j^{2}} \\
\mathscr{A}_{2 n+1}(0)=-2 q^{3 n^{2}+4 n+1} \sum_{j=0}^{n} q^{-j^{2}-j}+2 q^{3 n^{2}+2 n} \sum_{j=0}^{n-1} q^{-j^{2}-j} \\
\mathscr{A}_{2 n}(1)=-2 q^{3 n^{2}-2 n}\left(1-q^{4 n}\right) \sum_{j=0}^{n-1} q^{-j^{2}-j} \\
\mathscr{A}_{2 n+1}(1)=q^{3 n^{2}+n}\left(1-q^{4 n+2}\right) \sum_{|j| \leq n} q^{-j^{2}}
\end{gathered}
$$

$$
\mathscr{A}_{2 n}(2)=\frac{1}{1-q}\left(q^{3 n^{2}+n} \sum_{|j| \leq n} q^{-j^{2}}+2 q^{3 n^{2}+2 n} \sum_{j=0}^{n-1} q^{-j^{2}-j}\right),
$$

$$
\mathscr{A}_{2 n+1}(2)=\frac{-1}{1-q}\left(2 q^{3 n^{2}+4 n+1} \sum_{j=0}^{n} q^{-j^{2}-j}+q^{3 n^{2}+5 n+2} \sum_{|j| \leq n} q^{-j^{2}}\right) \text {. }
$$

LEMMA 12. $\left(\mathscr{A}_{n}(i), \mathscr{B}_{n}(i)\right)$ form a Bailey pair for $i=0,1, a=1$ and for $i=2$, $a=q$.

Proof. By (2.7) with $a=1$, the other half of the Bailey pair for $\mathscr{B}_{n}(0)$ (with $a=1)$ is

$$
\begin{aligned}
(1- & \left.q^{2 n}\right) \sum_{j=0}^{n} \frac{(q)_{n+j-1}}{(q)_{n-j}}(-1)^{n-j} q^{\left(n_{2}^{-j}\right) \mathscr{B}_{j}}(0) \\
& =\left(1-q^{2 n}\right) \sum_{j=0}^{n}\left[\begin{array}{l}
n+j \\
n-j
\end{array}\right] \frac{(q)_{j}}{\left(1-q^{n+j}\right)}(-1)^{n-j} q^{\left(\frac{n-j}{2}\right)} \\
& =V_{n} \quad(\text { by }(7.5)) \\
& =(-1)^{n} 2 q^{\left(n_{2}^{n+1}\right)}-\left(1-q^{n}\right) q^{2 n-2} U_{n-1} \quad \text { (by (7.8)) } \\
& =\mathscr{A}_{n}(0) .
\end{aligned}
$$

The formulas for $\mathscr{A}_{n}(0)$ given by (7.16) and (7.17) follow immediately from Lemma 11. Hence the $i=0$ case is established.

By (2.7) with $a=1$, the other half of the Bailey pair for $\mathscr{B}_{n}(1)($ with $a=1)$ is

$$
\begin{aligned}
(1- & \left.q^{2 n}\right) \sum_{j=0}^{n} \frac{(q)_{n+j-1}}{(q)_{n-j}}(-1)^{n-j} q^{\left(n^{n-j}\right)} \mathscr{B}_{j}(1) \\
& =\left(1-q^{2 n}\right) \sum_{j=1}^{n}\left[\begin{array}{c}
n+j-1 \\
n-j
\end{array}\right](q)_{j-1}(-1)^{n-j} q^{(n-j)} \\
& =\left(1-q^{2 n}\right) U_{n} \quad(\text { by }(7.4)) \\
& =\mathscr{A}_{n}(1),
\end{aligned}
$$

where the formulas for $\mathscr{A}_{n}(1)$ given by (7.18) and (7.19) follow directly from Lemma 11. Hence the case $i=1$ is established. 
Finally by (2.7) with $a=q$, the other half of the Bailey pair for $\mathscr{B}_{n}(2)$ (with $a=q)$ is

$$
\begin{aligned}
& \frac{\left(1-q^{2 n+1}\right)}{(1-q)} \sum_{j=0}^{n} \frac{(q)_{n+j}}{(q)_{n-j}}(-1)^{n-j} q^{\left(\frac{n-j}{2}\right)} \mathscr{B}_{j}(2) \\
& \quad=\frac{\left(1-q^{2 n+1}\right)}{(1-q)} \sum_{j=0}^{n}\left[\begin{array}{c}
n+j+1 \\
n-j
\end{array}\right] \frac{(q)_{j}}{1-q^{n+j+1}}(-1)^{n-j} q^{\left(\frac{n-j}{2}\right)} \\
& \quad=\frac{1}{1-q} \sum_{j=0}^{n}\left[\begin{array}{c}
n+j+1 \\
n-j
\end{array}\right](q)_{j} \frac{\left(1-q^{n+j+1}+q^{n+j+1}\left(1-q^{n-j}\right)\right)}{\left(1-q^{n+j+1}\right)}(-1)^{n-j} q^{\left(\frac{n-j}{2}\right)} \\
& =\frac{1}{1-q}\left(\sum_{j=0}^{n}\left[\begin{array}{c}
n+j+1 \\
n-j
\end{array}\right](q)_{j}(-1)^{n-j} q^{\left(\frac{n-j}{2}\right)}\right. \\
& \left.\quad+\sum_{j=0}^{n}\left[\begin{array}{c}
n+j \\
n-j-1
\end{array}\right](q)_{j}(-1)^{n-j} q^{\left(\frac{n-j}{2}\right)+n+j+1}\right) \\
& =\frac{1}{1-q}\left(U_{n+1}-q^{2 n} U_{n}\right)=\mathscr{A}_{n}(2),
\end{aligned}
$$

where the formulas for $\mathscr{A}_{n}(2)$ given by (7.20) and (7.21) follow directly from Lemma 11. Hence the case $i=2$ is established.

We are now set for our main result on the seventh order mock theta functions, which does indeed link these functions with Hecke type series involving the number 7.

\section{THEOREM 13.}

$$
\begin{aligned}
\mathscr{F}_{0}(q) \equiv & \sum_{n=0}^{\infty} \frac{q^{n^{2}}}{\left(q^{n+1}\right)_{n}} \\
= & \frac{1}{(q)_{\infty}}\left(\sum_{\substack{n=0 \\
|j| \leq n}} q^{7 n^{2}+n-j^{2}}\left(1-q^{12 n+6}\right)\right. \\
& \left.\quad-2 q \sum_{n=0}^{\infty} \sum_{j=0}^{n} q^{7 n^{2}+8 n-j^{2}-j}\left(1-q^{12 n+13}\right)\right),
\end{aligned}
$$

$$
\begin{aligned}
\mathscr{F}_{1}(q) \equiv \sum_{n=1}^{\infty} \frac{q^{n^{2}}}{\left(q^{n}\right)_{n}} & \frac{1}{(q)_{\infty}}\left(-2 \sum_{n=0}^{\infty} \sum_{j=0}^{n-1} q^{7 n^{2}-2 n-j^{2}-j}\left(1-q^{4 n}\right)\right. \\
& \left.+\sum_{\substack{n=0 \\
|j| \leq n}} q^{7 n^{2}+5 n+1-j^{2}}\left(1-q^{4 n+2}\right)\right),
\end{aligned}
$$




$$
\begin{aligned}
\mathscr{F}_{2}(q) \equiv \sum_{n=0}^{\infty} \frac{q^{n^{2}+n}}{\left(q^{n+1}\right)_{n+1}} \\
=\frac{1}{(q)_{\infty}}\left(\sum_{\substack{n=0 \\
|j| \leq n}}^{\infty} q^{7 n^{2}+n-j^{2}}\left(1-q^{8 n+3}\right)\right. \\
\left.\quad-2 q^{2} \sum_{n=0}^{\infty} \sum_{j=0}^{n} q^{7 n^{2}+8 n-j^{2}-j}\left(1-q^{8 n+7}\right)\right) .
\end{aligned}
$$

Proof. For $i=0,1$, we have

$$
\mathscr{F}_{i}(q)=\sum_{n=0}^{\infty} q^{n^{2}} \mathscr{B}_{n}(i)=\frac{1}{(q)} \sum_{n=0}^{\infty} q^{n^{2}} \mathscr{A}_{n}(i),
$$

and equations (7.22) and (7.23) now follow by substituting the formulae for $\mathscr{A}_{n}(i)$ from (7.16)-(7.19) into this identity.

For $i=2, a=q$, we have

$$
\mathscr{F}_{2}(q)=\sum_{n=0}^{\infty} q^{n^{2}+n} \mathscr{B}_{n}(2)=\frac{1}{\left(q^{2}\right)_{\infty}} \sum_{n=0}^{\infty} q^{n^{2}+n} \mathscr{A}_{n}(2),
$$

and (7.24) follows from (7.21) and (7.22).

8. Conclusion. Clearly we are just beginning to understand Ramanujan's last brilliant creation: the Mock Theta Functions. It is my hope that these discoveries will assist in proving many of the results on fifth order functions that are given in his "Lost" Notebook [5]. Also is there significant arithmetic information about sums of three squares in (5.16)?

Finally we suggest a further study of the Bailey pair $\left(\alpha_{n}(b), \beta_{n}(b)\right)$, where $\beta_{n}(b)=(b q)_{n} /(q)_{2 n}$. The first few $\alpha_{n}(b)$ are

$$
\begin{aligned}
& \alpha_{0}(b)=1, \\
& \alpha_{1}(b)=-b q-b, \\
& \alpha_{2}(b)=b^{2} q^{3}+b q^{4}+b q^{3}-q^{2}, \\
& \alpha_{3}(b)=-b^{3} q^{6}-b^{2} q^{8}-b^{2} q^{7}-b^{2} q^{6}-b q^{8}+b q^{5}+q^{7}+q^{5} .
\end{aligned}
$$

Furthermore $\sum_{n \geq 0} q^{n^{2}} \beta_{n}(b)$ is an important theta series for $b=0, q^{-1 / 2},-1$, and is the first seventh order mock series for $b=1$. Can a representation of $\alpha_{n}(b)$ be found that directly yields these facts as special cases?

\section{REFERENCES}

1. G. E. Andrews, On basic hypergeometric series, mock theta functions and partitions. I, Quart. J. Math. Oxford Ser. 17 (1966), 64-80.

2. , On basic hypergeometric series, mock theta functions and partitions. II, Quart. J. Math. Oxford Ser. 17 (1966), 132-143.

3. , On the theorems of Watson and Dragonette for Ramanujan's mock-theta functions, Amer. J. Math. 88 (1966), 454-490.

4. The theory of partitions, Encyclopedia of Math. and Its Applications, Vol. 2 (G.-C. Rota, ed.), Addison-Wesley, Reading, Mass., 1976. 
5. ___ An introduction to Ramanujan's "lost" notebook, Amer. Math. Monthly 86 (1979), 89-108.

6. Connection coefficient problems and partitions, Proc. Sympos. Pure Math., vol. 34, Amer. Math. Soc., Providence, R. I., 1979, pp. 1-24.

7. __ Hecke modular forms and the Kac-Petersen identities, Trans. Amer. Math. Soc. 283 (1984), $451-458$.

8. __ Multiple series Rogers-Ramanujan type identities, Pacific J. Math. 114 (1984), 267-283.

9. W. N. Bailey, Identities of the Rogers-Ramanujan type, Proc. London Math. Soc. (2) 50(1949), 1-10.

10. D. M. Bressoud, Hecke modular forms and q-Hermite polynomials (to appear).

11. L. A. Dragonette, Some asymptotic formulae for the mock theta series of Ramanujan, Trans. Amer. Math. Soc. 72 (1952), 474-500.

12. E. Hecke, Über einen Zusammenhang zwischen elliptischen Modulfunktionen und indefiniten quadratischen Formen, Mathematische Werke, Vandenhoeck und Ruprecht, Göttingen, 1959, pp. 418-427.

13. V. G. Kac and D. H. Peterson, Affine Lie algebras and Hecke modular forms, Bull. Amer. Math. Soc. (N.S.) 3 (1980), 1057-1061.

14. S. Ramanujan, Collected papers, Cambridge Univ. Press, London, 1927; Reprinted, Chelsea, New York, 1962.

15. L. J. Rogers, Second memoir on the expansion of certain infinite products, Proc. London Math. Soc. 25 (1894), 318-343.

16. D. Shanks, $A$ short proof of an identity of Euler, Proc. Amer. Math. Soc. 2 (1951), 747-749.

17. , Two theorems of Gauss, Pacific J. Math. 8 (1958), 609-612.

18. L. J. Slater, Generalized hypergeometric functions, Cambridge Univ. Press, London, 1966.

19. G. N. Watson, The final problem: an account of the mock theta functions, J. London Math. Soc. 11 (1936), 55-80.

20. The mock theta functions. II, Proc. London Math. Soc. (2) 42 (1937), 272-304.

21. A A note on Spence's logarithm transcendant, Quart. J. Math. Oxford Ser. 8 (1937), 39-42.

22. D. B. Sears, On the transformation theory of basic hypergeometric functions, Proc. London Math. Soc. (2) 53 (1951), 158-180.

Department of Mathematics, The Pennsylvania State University, University Park, PennsylVANIA 16802 